\title{
Joint Detection for Image Transmission Based on Schur Algorithm over Wireless Sensor Network
}

\author{
Yanjun HU \\ Key Laboratory of Intelligent Computing and Signal Processing, Ministry of Education, \\ Anhui University, Hefei, China \\ E-mail: yanjunhu@ahu.edu.cn \\ Received August 3, 2009; accepted September 1, 2009
}

\begin{abstract}
To achieve much efficient multimedia transmission over an error-prone wireless network, there are still some problem must to be solved, especially in energy limited wireless sensor network. In this paper, we propose a joint detection based on Schur Algorithm for image wireless transmission over wireless sensor network. To eliminate error transmissions and save transmission energy, we combine Schur algorithm with joint dynamic detection for wireless transmission of JPEG 2000 encoded image which we proposed in [1]. Schur algorithm is used to computing the decomposition of system matrix to decrease the computational complexity. We describe our transmission protocol, and report on its performance evaluation using a simulation testbed we have designed for this purpose. Our results clearly indicate that our method could approach efficient images transmission in wireless sensor network and the transmission errors are significantly reduced when compared to regular transmissions.
\end{abstract}

Keywords: Joint Detection, Image Wireless Transmission, Schur Algorithm, Sensor Network

\section{Introduction}

In recent years, wireless multimedia communication systems have become increasingly popular as an inexpensive and promising means for ubiquitous and wireless communications. The demand of Multimedia services is growing for wireless units. The wireless imaging is expected to become a breakthrough application for the successful commercial development of wireless communication systems. In order to be suitable for wireless multimedia applications, Wireless multimedia sensor networks are concerned more and more. However, to achieve much efficient multimedia transmission over an error-prone wireless network, there are still some problem must to be solved, especially in energy limited wireless sensor network. Wireless sensor networks are an enabling technology for many future applications, but still some challenges in wireless sensor network [2].

JPEG recently developed a new kind of technology which referred to as JPEG 2000 Wireless (JPWL) [3,4] to achieve efficient transmission of JPEG 2000 images over an error-prone wireless transmission environment. More specifically, JPWL extends the elements in the core coding system with mechanisms for error protection

*The work is supported by National Natural Science Foundation (No.60772123) and the Scientific Research Foundation for the Returned Overseas Chinese Scholars, State Education Ministry. and correction. It supports a set of tools for error protection and correction including Forward Error Correcting codes, Unequal Error Protection, data partitioning and interleaving, turbo codes, and robust arithmetic coding. Examples of tools envisioned to be supported by JPWL include the techniques presented in [5-15]. However, JPWL is not linked to a specific network or transport protocol. It merely provides a generic solution for the robust transmission of JPEG 2000 images over error-prone networks. It is open to users and manufacturers. Especially in wireless sensor network, there are still some problems need to explore. There are a few of research about the application of JPEG2000 in wireless sensor. Reference [16] proposed an energy efficient JPEG 2000 image transmission system over point-topoint wireless sensor networks. Reference [17] proposed a scheme of multiple bitstream image transmission over wireless sensor networks, it used multiple bitstream images encoding to achieve error robust transmission and small fragment burst transmission to achieve efficient transmission. References $[18,19]$ gives different method of distributed JPEG2000 Image Compression for Sensor Networks. Most research concerning wireless image transmission has not considered the physical properties of wireless channels such as multipath propagation, multiple-access interference, etc. Therefore, in Reference [1], considering the properties of wireless channel, we have 
proposed a joint dynamic detection method for receiver from the view of physical layer.

The Schur algorithm is more amendable to a finegrained parallel implementation with systolic processor arrays in computing of system matrix. Only Reference [20] derives a generalized Schur algorithm for joint-detection in TD-CDMA mobile radio systems. To eliminate error transmissions and save transmission energy, we combine Schur algorithm with a joint dynamic detection for wireless transmission of JPEG 2000 encoded image which we proposed in [1]. Schur algorithm is used to computing the decomposition of system matrix to decrease the computational complexity. A joint detection method for image wireless transmission based on Schur Algorithm over wireless sensor network is proposed. We describe our transmission protocol, and report on its performance evaluation using a simulation testbed we have designed for this purpose. Our results clearly indicate that our method could approach efficient images transmission in wireless sensor network and the transmission errors are significantly reduced when compared to regular transmissions.

This paper is organized as follows: in Section 2 we provide the model of wireless transmission system. In Section 3, we propose a joint detection based on Schur algorithm. Simulation results are showed in Section 4.

\section{Image Wireless Transmission System Model}

In sensor field of wireless sensor network, data collected by sensors are transmitted to a sink, as showed in Figure 1. The data collection sensors are typically subjected to strict energy constraints. The data collection nodes collect data on a physical phenomenon that is of interest and communicate them to the sink node over a wireless link which performs required joint processing. The low-cost sensors only are responsible for images collection. The sink node is responsible for the image gathering and error detection. The sink node is less energy-constrained compared to the sensing nodes. It does not have any energy constrains attached to it. Most of the complex processing is thus carried out at the sink node resulting in better energy efficiency at the energy constrained sensor node level.

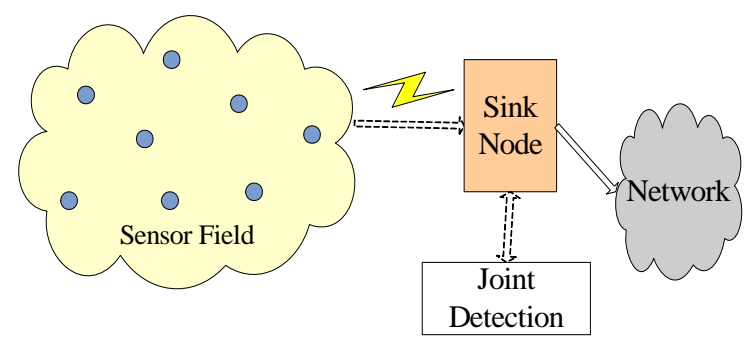

Figure 1. Wireless sensor networks transmission architecture.
The system model is shown in Figure 2. In the transmitter sensor, the image signals are first compressed and farmed into a certain number of layers by a general JPEG2000 encoder. Then the compressed data is jointly coded. After the carrier has been modulated, the data is transmitted through wireless channels. In the receiver, the signal is first demodulated. Then joint detection is used to reduce interferences and noise based schur algorithm before JPEG2000 decompression. After joint detection, the image data could be decompressed to redisplay in the sink receiver or send to other network directly.

The sink node performs periodic polling of data collection nodes, at any given time the sink node itself may request simultaneous data transmissions from a set of chosen nodes.

\section{Joint Detection Based on Schur Algorithm}

Considering the physical properties of wireless communication channels, either interference cancellation or multiuser detection is applied at the receiver side so as to reduce the load for error correction and control. Interference cancellation and error recovery are jointly considered.

We assume that there are $N$ transmitting sensor nodes and each has $b_{i}$ bits to transmit. All sending signals could be written as vector form $\boldsymbol{b}_{N \times 1}=\left(b_{1}, b_{2} \cdots b_{N}\right)^{T}$, after modulation, the $N \times N$ diagon-allized vector $\operatorname{diag}\left(b_{1}, b_{2} \cdots b_{N}\right)$ multiplies with modulation signal $\mathbf{C}_{N \times N}$. The output signal of transmitter is denoted with vector matrix $\boldsymbol{A}_{N \times 1}=\left[\begin{array}{llll}a_{1} & a_{2} & \cdots & a_{N}\end{array}\right]^{T}$.

The sending signal is transmitted through wireless channel, the input signal of sink receiver is represented as $\boldsymbol{D}_{N \times 1}$

$$
\boldsymbol{D}_{N \times 1}=\left[\begin{array}{llll}
d_{1} & d_{2} \cdots d_{N}
\end{array}\right]^{T}=\boldsymbol{A}_{N \times 1}+\boldsymbol{n}_{N \times 1}
$$

where $n_{N \times 1}$ is noise vector.

In asynchronous cause, the elements of above received signal matrix $\boldsymbol{A}_{N \times 1}$ only simply are shifted according to different delay correspondingly before are summed.

The output of demodulator is:

$$
\boldsymbol{Y}=C_{K \times N} D_{N \times 1}=C_{K \times N} A_{N \times 1}+z_{k \times 1}
$$

where $z_{k \times 1}=C_{K \times N} n_{N \times 1}$

It can also be written in vector form as (3)

$$
\boldsymbol{Y}=\boldsymbol{R} \boldsymbol{W} \boldsymbol{b}+\boldsymbol{Z}
$$

where $\boldsymbol{R}$ is channel crosscorrelation matrix vector, and $\boldsymbol{W}$ is signal power matrix. Let $\boldsymbol{W}$ normalized to diagonal unit matrix, then we have

$$
\boldsymbol{Y}=\boldsymbol{R} \boldsymbol{b}+\boldsymbol{Z}
$$






Figure 2. Proposed system model of image wireless transmission.

Decomposing the matrix $\boldsymbol{R}=\boldsymbol{U T}, \boldsymbol{U}$ is $N \times N$ matrix with orthogonal column, and $\boldsymbol{T}$ is an upper triangular matrix. Then the receiving signal be written as

$$
\tilde{\boldsymbol{Y}}=\boldsymbol{U}^{\boldsymbol{H}} \boldsymbol{Y}=\boldsymbol{T} \boldsymbol{b}+\eta
$$

where $\eta=\boldsymbol{U}^{H} \boldsymbol{Z}$.

We can solve the sending signal for bottom to up one by one according to the upper triangular characteristic of coefficient matrix. It can be used to find the vector $\mathbf{b}_{N \times 1}$ that minimizes $\|\mathbf{y}-\mathbf{H b}\|_{2}^{2}$ by solving the triangular system of equations $\mathbf{R} \mathbf{b}=\mathbf{U}^{H} \mathbf{y}$.

To obtain rapid matrix solution, the Schur algorithm is be used to decomposing $\boldsymbol{R}=\boldsymbol{U T}$. The Schur algorithm is a way to efficiently compute the $\boldsymbol{U} \boldsymbol{T}$ decomposition of a Toeplitz structured matrix.

In order to realize our method of joint error detection, we used same structure and joint method which we proposed in Reference [1], and combine it with Schur algorithm. It preserves the same codestream structure as the one defined by the JPEG2000 core coding standard. Hence, even if the decoder has no knowledge of encode embedding, the resulting codestream can also be decoded using the decoder of JPEG2000 core coding system and conforms to the JPEG2000. Namely, the bit stream is compatible with JPEG2000.

\section{Simulation Results}

Based on the method of joint detection that described as above, we made simulation experiment on microcomputer system. There are 20 collection sensors in the cluster, and one is image collection node, others are data collection nodes. DS-CDMA base-band model is employed as a wireless transmission system in evaluating the performance of the proposed method. A set of spread-spectrum Gold sequences of length 127 is chosen

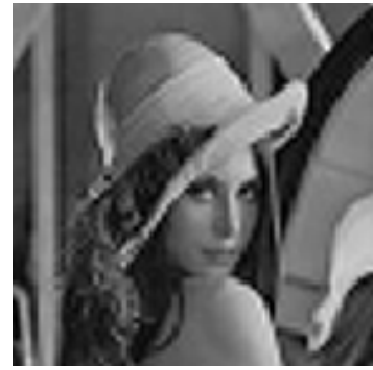

(a) Original image



(c) Conventional received $\mathrm{BER}=3.82 \times 10^{-1}$

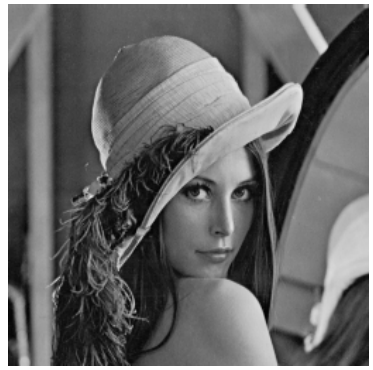

(b)Wavelet compression

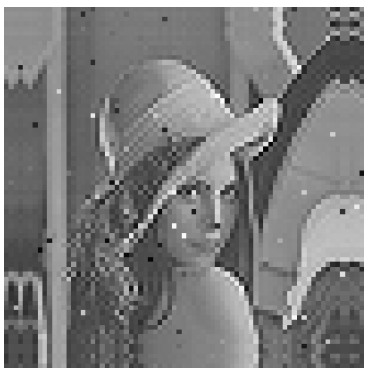

(d) Detection of Reference [1] $\mathrm{BER}=6.83 \times 10^{-2}$

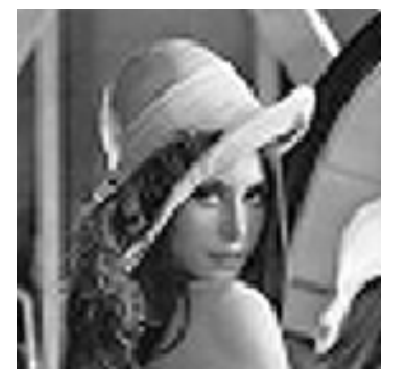

(e) Joint detection of this paper $\mathrm{BER}=8.41 \times 10^{-3}$

Figure 3. Image transmitted (example 1: $\mathrm{SNR}=4 \mathrm{~dB}$, nodes=20). 
and an ideal power controlled environment with a two paths Rayleigh multipath fading channel is assumed. The signal-to-noise ratio (SNR) can be variable in the simulation. In our simulating experiments, the size of the image is $256 \times 256$ pixels, while the gray level is 8 bits/pixels, when compressed by the JPEG2000 verification model.

Performance can be evaluated by comparing simulation results. In Figures 3,4, we report the results we have obtained with 10 and 20 users in the system and SNR equal to $6 \mathrm{~dB}$ and $4 \mathrm{~dB}$ respectively. Figure 3(a) and Figure 4(a) are the original JPEG image in the transmitter respectively. Figure 3(b) and Figure 4(b) are the JPEG2000 coded images with wavelet compression; Figure 3(c) and Figure 4(c) are the decompression images in the receiver without joint detection. As you can see, the pictures are not particularly clear. This is mainly due to the noise and interference that wireless communication experiences. Figure 3(d) and Figure 4 (d) show the results we obtained for the decompression images at the sink receiver where the joint detection which we proposed in Reference [1] is used. Figure 3(e) and Figure 4(e) show the results we obtained for the



(a) Original image

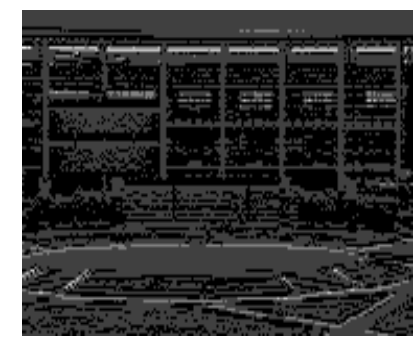

(c) Conventional received $\mathrm{BER}=9.55 \times 10^{-1}$

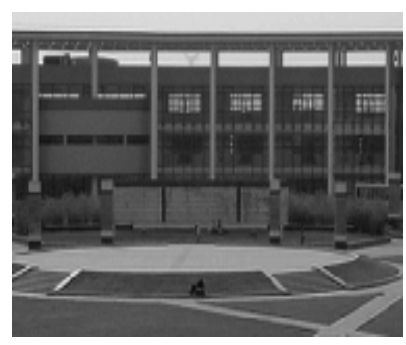

(e) Joint detection of this paper $\mathrm{BER}=1.23 \times 10^{-2}$

Figure 4. Image transmitted (example 2: $\mathrm{SNR}=-5 \mathrm{~dB}$, user number=20).



Figure 5. Given user number $K=20$, the bit error rates with different SNR.

decompression images at the receiver where the joint detection which proposed in this paper is used. As we can see, the quality of the images is better than that seen in Figure 3(d) and Figure 4(d). The error probability comparisons with different SNR are showed in Figure 5. As shown, our method decreased the error probability and increased the image quality compare to the method of reference, particularly in noise channel with low signal and noise ratio.

The results prove that using joint detection we can obtain higher image quality. Interference and transmission errors are significantly reduced.

\section{Conclusions}

The proposed method of joint detection based on Schur Algorithm for image wireless transmission over wireless sensor network has been described above. It protects encoded images against error and improves the performance of wireless image transmission. Simulation results show that our method could approach efficient images transmission in wireless sensor network and the transmission errors are significantly reduced when compared to regular transmissions.

In the future, we plan to extend our simulation experiment, and further investigate our transmission scheme for wireless multimedia sensor network based applications. Further extension is under study.

\section{References}

[1] Y. J. Hu, A. Boukerche, and R. W. N. Pazzi, “An efficient joint dynamic detection technique for wireless transmission of JPEG2000 encoded images,” Proceeding of the 2006 International Conference on Communications and Mobile Computing IWCMC'06, Vancouver, Canada, pp. 395-400, July 2006. 
[2] I. F. Akyildiz, W. Su, Y. Sankarasubramaniam, and E. Cayirci, “A survey on sensor networks," IEEE Communications Magazine, pp. 102-114, August 2002.

[3] A. Skodras, C. Christopoulos, and T. Ebrahimi, "The JPEG 2000 still image compression standard,” IEEE Signal Processing Magazine, Vol. 18, No. 5, pp. 36-58, September 2001.

[4] "JPEG 2000 image coding system-Part 11: Wireless JPEG 2000-working draft version 3.1,” ISO/IEC JTC1/ SC29/WG1 WG1N3294, April, 2004.

[5] A. Natu and D. Taubman, "Unequal protection of JPEG 2000 codestreams in wireless channels," in IEEE Proceedings Globecom'02, Taipei, Taiwan, Vol. 1, pp. 534-538, November 2002,.

[6] D. Nicholson, C. Lamy-Begot, X. Naturel, and C. Poulliat, "JPEG 2000 backward compatible error protection with Reed-Solomon codes," IEEE Transactions on Consumer Electronics, Vol. 49, No. 4, pp. 855-860, November 2003.

[7] F. Frescura, M. Giorni, C. Feci, and S. Cacopardi, "JPEG 2000 and MJPEG 2000 transmission in 802.11 wireless local area networks," IEEE Transactions on Consumer Electronics, Vol. 49, No. 4, pp. 861-871, November 2003.

[8] M. Grangetto, E. Magli, and G. Olmo, "Error sensitivity data structures and retransmission strategies for robust JPEG 2000 wireless imaging," IEEE Transactions on Consumer Electronics, Vol. 49, No. 4, pp. 872-882, November 2003.

[9] K. Munadi, M. Kurosaki, K. Nishikawa, and H. Kiya, "Error protection for JPEG2000 encoded images and its evaluation over OFDM channel, ISCAS’03,” Proceedings of the 2003 International Symposium on Circuits and Systems, Vol. 2, pp. II-432-II-435, May 2003.

[10] K. Munadi, M. Kurosaki, K. Nishikawa, and H. Kiya, “A robust error protection technique for JPEG2000 codestream and its evaluation in CDMA environment, APCC 2003,” The 9th Asia-Pacific Conference on Communications, Vol. 2, pp. 654-658, September 21-24, 2003.

[11] V. Chande and N. Farvardin, "Progressive transmission of images over memoryless noisy channels," IEEE Journal on Selected Areas in Communications, Special Issue on Error Resilient Coding, Vol. 18, No. 6, pp. 850-861, June 2000.

[12] A. E. Mohr, E. A. Riskin, and R. E. Ladner, "Unequal loss protection: Graceful degradation of image quality over packet erasure channels through forward error cor- rection,” IEEE Journal on Selected Areas in Communications, Vol. 18, No. 7, pp. 819-828, 2000.

[13] Y. Charfi, R. Hamzaoui, and D. Saupe, "Model-based real-time progressive transmission of images over noisy channels," IEEE Wireless Communications and Networking, WCNC 2003, Vol. 2, pp. 784-789, March 2003.

[14] Sanchez and Mandal, "Robust transmission of Jpeg2000 images over noisy channels," IEEE Transactions on Consumer Electronics, Vol. 48, No. 3, pp. 451-456, August 2002.

[15] A. Boukerche and Y. J. Hu, "QoS-based JPEG images transmission protocol for wireless CDMA communication systems, international workshop on modeling analysis and simulation of wireless and mobile systems," Proceedings of the 1st ACM Workshop on Wireless Multimedia Networking and Performance Modeling, pp. 3845, Montreal, Quebec, Canada, 2005.

[16] W. Yu, Z. F. Sahinoglu, and A. Vetro, "Energy efficient JPEG 2000 image transmission over wireless sensor networks,” IEEE Global Telecommunications Conference (GLOBECOM), Dallas, Tex, Vol. 5, pp. 2738-2743, USA.

[17] M. Wu and C. W. Chen, "Multiple bitstream image transmission over wireless sensor networks,” Proceedings of IEEE Sensors 2003, Second IEEE International Conference on Sensors, Vol. 2, pp. 727-731, Toronto, Canada, October 22-24, 2003.

[18] H. Wu and A. A. Abouzeid, "Energy efficient distributed JPEG2000 image compression in multihop wireless networks,” Proceedings of the 4thWorkshop on Applications and Services in Wireless Networks (ASWN'04), Boston, Mass, USA, pp. 152-160, August 2004.

[19] R. Wagner, R. Nowak, and R. Baraniuk, “Distributed image compression for sensor networks using correspondence analysis and super-resolution,” Proceedings of the IEEE International Conference on Image Processing (ICIP’03), Barcelona, Spain, Vol. 1, pp. 597-600, September 2003.

[20] M. Vollmer, M. Haardt, and J. Gotze, "Schur algorithms for joint detection in TD-CDMA based mobile radio systems," Annals of Telecommunications, Vol. 54, No. 7-8, July-August 1999. 